\title{
7. Ehrenamtliches Engagement von Frauen und Männern im Verlauf der zweiten Lebenshälfte
}

\author{
Claudia Vogel und Laura Romeu Gordo
}

\section{Kernaussagen}

Für Frauen und Männer steigt die Wahrscheinlichkeit, sich in formalen Organisationen ehrenamtlich zu engagieren, zunächst an, sinkt dann mit zunehmendem Alter jedoch wieder: Im Alter von 40 bis 58 Jahren wird es wahrscheinlicher, sich ehrenamtlich zu engagieren. Ab 59 Jahren wird es mit steigendem Alter unwahrscheinlicher, ehrenamtlich engagiert zu sein. Am wahrscheinlichsten ist die ehrenamtliche Partizipation im Alter zwischen 53 und 63 Jahren, in dieser Lebensphase liegt sie über 19 Prozent.

Über den gesamten Verlauf der zweiten Lebenshälfte haben Männer eine höhere Wahrscheinlichkeit, sich ehrenamtlich zu engagieren, als Frauen: Diese Geschlechterunterschiede sind zu Beginn der zweiten Lebenshälfte größer als im höheren Alter. Das heißt, die Geschlechterunterschiede verringern sich mit steigendem Alter insbesondere in der Ruhestandsphase.

Die Wahrscheinlichkeit für Frauen und Männer, ehrenamtlich engagiert zu sein, ist für später geborene Kohorten über den Alternsverlauf von 40 bis 90 Jahren höher als für früher geborene. Die Geschlechterunterschiede im Ehrenamt bleiben in allen Kohorten bestehen: Während die Kohorten einen ähnlichen Alternsverlauf aufweisen, scheint sich der Wendepunkt, ab dem die Ehrenamtswahrscheinlichkeit mit steigendem Alter sinkt, nach hinten zu verschieben. Dadurch, dass Frauen ähnliche Entwicklungen im Ehrenamt über die Kohorten hinweg aufweisen wie Männer, ist bislang keine Verringerung des Geschlechterunterschieds für später geborene Kohorten zu beobachten.

Die Wahrscheinlichkeiten der Neuaufnahme und Beendigung von ehrenamtlichem Engagement unterscheiden sich im Alternsverlauf, es gibt jedoch kaum Geschlechterunterschiede: Die Wahrscheinlichkeit, eine ehrenamtliche Tätigkeit neu aufzunehmen, nimmt bis zu einem Alter von 54 Jahren zu, und sie sinkt dann mit dem Älterwerden ab. Die Beendigung von Ehrenamt bleibt hingegen im Alternsverlauf stabil. Die stetig geringere Wahrscheinlichkeit, im höheren Alter einer ehrenamtlichen Tätigkeit nachzugehen, ergibt sich daraus, dass in diesem höheren Lebensalter zunehmend weniger Personen ein Ehrenamt neu anfangen und gleichzeitig nicht weniger Engagierte ihre ehrenamtlichen Tätigkeiten beenden. Prozesse der Aufnahme von Ehrenämtern unterscheiden sich nicht signifikant nach Geschlecht und Beendigungsprozesse unterscheiden sich nur minimal nach Geschlecht. 


\subsection{Einleitung}

Viele Ältere sind heute trotz beruflichen Ruhestandes häufig aktiv und übernehmen eine Vielzahl von produktiven Tätigkeiten in der Familie, der Nachbarschaft und der Zivilgesellschaft. Dies können Hausarbeit, Enkelkinderbetreuung, Pflegetätigkeiten, Alltagsunterstützung oder Ehrenämter sein. Solche Tätigkeiten sind für die Wohlfahrtsproduktion und die Lebensqualität älterer Menschen in Deutschland sehr bedeutsam (siehe Kapitel 6 Unbezahlte Sorgetätigkeiten in diesem Band). Ein ehrenamtliches Engagement in einem Verein oder einer Organisation bringt für die Engagierten vielerlei Vorteile mit sich. Es kann sinnstiftend sein und das Gefühl vermitteln, gebraucht zu werden und mitbestimmen zu können. Es kann zudem Freude bereiten und den Alltag strukturieren. Auch geht ehrenamtliches Engagement mit der Knüpfung und Pflege sozialer Kontakte einher, sowie mit der Möglichkeit, die eigenen Erfahrungen und Kenntnisse einzubringen und neue Kenntnisse und Fertigkeiten zu erwerben. Ein Ehrenamt ermöglicht ferner sozial teilzuhaben, und es bringt auch Anerkennung mit sich - gerade mit steigendem Alter, wenn der Beruf in den Hintergrund gerät, sind dies keine unwichtigen Aspekte. Belegt ist darüber hinaus, dass Personen, die sich engagieren, zufriedener und gesünder sind als Personen, die sich nicht ehrenamtlich oder freiwillig engagieren (zum Forschungsstand vgl. Simonson \& Vogel 2018/im Erscheinen).

Wie entwickelt sich das ehrenamtliche Engagement von Menschen, wenn sie das mittlere Lebensalter erreicht haben und älter werden? Aus Querschnittsvergleichen der Anteile Engagierter lässt sich ablesen, dass Frauen und Männer in den Altersgruppen von 40 bis 69 Jahren überdurchschnittlich häufig engagiert sind. Im Alter ab 70 Jahren sind die Anteile Engagierter geringer (und ab 75 Jahre deutlich geringer) (vgl. Vogel, Hagen, Simonson, \& Tesch-Römer 2017: 104). Sind im höheren Alter nur Menschen engagiert, die ein früher im Lebenslauf begonnenes Engagement bis ins Alter fortsetzen oder planen auch ältere Men- schen, die bislang nicht engagiert waren, ein Ehrenamt aufzunehmen? Befunde des Deutschen Alterssurveys (DEAS) 2014 belegen, dass Menschen auch in der zweiten Lebenshälfte noch ehrenamtliche Tätigkeiten neu aufnehmen, denn von den gut 22 Prozent der im Jahr 2014 ehrenamtlich engagierten Personen im Alter von 40 bis 85 Jahren hat „ein Drittel seit 2009 ein (zusätzliches) Engagement begonnen" (vgl. Wetzel \& Simonson 2017: 90). Laut Ergebnissen des Deutschen Freiwilligensurveys haben sich lediglich 35 Prozent der Personen über 64 Jahren rückblickend über ihren gesamten Lebenslauf nie engagiert (Müller \& Tesch-Römer 2017: 164).

Gleichzeitig beenden viele Personen ihr ehrenamtliches Engagement im höheren Alter. Gründe hierfür liegen zum einen bei den ehrenamtlichen Tätigkeiten selbst. Entweder war eine solche Tätigkeit von vornherein zeitlich begrenzt, was rund die Hälfte der 65-Jährigen und Älteren als Beendigungsgrund angibt, oder aber der zeitliche Aufwand durch das Engagement wurde als zu hoch empfunden (dies trifft auf rund ein Drittel der 65-Jährigen und Älteren zu, vgl. Müller \& TeschRömer 2017: 162-163). In anderen Fällen hatten die Engagierten ein Lebensalter erreicht, das als Altersgrenze für das spezifische Engagement gilt, bei der Freiwilligen Feuerwehr liegt diese Altersgrenze z. B. in der Regel bei 65 Jahren (zu Altersgrenzen im Ehrenamt vgl. Künemund \& Vogel 2018). Das Erreichen einer Altersgrenze als Beendigungsgrund trifft auf 38 Prozent der 65-Jährigen und Älteren zu (hierbei waren Mehrfachnennungen erlaubt, das heißt es konnten mehrere Gründe für die Beendigung angegeben werden, vgl. Müller \& Tesch-Römer 2017: 162-63). Die Gründe, die dazu beitragen, dass Menschen im Lebenslauf ein ehrenamtliches Engagement beenden, können zum anderen auch in anderen Lebensbereichen liegen, z. B. werden von jeweils rund einem Drittel der 65-Jährigen und Älteren berufliche oder familiäre Gründe dafür genannt, dass sie ein früheres Engagement beenden mussten, und von rund ei- 
nem Viertel gesundheitliche Gründe. Darüber hinaus hat rund ein Drittel der 65-Jährigen und Älteren ein früheres Engagement beendet, weil sie keine Verpflichtungen mehr haben wollten (zu den Beendigungsgründen vgl. Müller \& Tesch-Römer 2017: 162-163).

\section{Alternsverläufe ehrenamtlichen Engagements von Frauen und Männern}

Wie verändern sich die Wahrscheinlichkeiten, sich ehrenamtlich zu engagieren, bei Frauen und Männern mit dem Älterwerden? Und unterscheiden sich die Alternsverläufe ehrenamtlichen Engagements von Frauen und Männern? Aus Querschnittsanalysen auf Basis des Deutschen Alterssurveys ist bekannt, dass Frauen im Alter von 40 bis 85 Jahren zu etwas geringeren Anteilen ehrenamtlich engagiert sind (19 Prozent im Jahr 2014) als Männer (26 Prozent im Jahr 2014) (vgl. Wetzel \& Simonson 2017: 87; für 1996 und 2002 vgl. Künemund 2006; für 2008 vgl. Naumann \& Romeu Gordo 2010). Allerdings ist der Geschlechterunterschied in der Engagementbeteiligung in verschiedenen Altersgruppen unterschiedlich ausgeprägt: Während die Engagementquote bei Frauen über 64 Jahren deutlich unter der Engagementquote der Männer dieser Altersgruppe liegt, sind Frauen im Alter zwischen 30 und 49 Jahren zu einem etwas höheren Anteil freiwillig engagiert als Männer (vgl. Vogel et al. 2017: 105).

Diese Befunde deuten darauf hin, dass das Engagement in bestimmten Lebensphasen biografisch mit spezifischen Lebenssituationen verknüpft ist, etwa dem Leben mit minderjährigen Kindern im Haushalt. In einer Lebenslaufbetrachtung könnte es sein, dass mit einer Veränderung der Lebenssituation eine Veränderung der ehrenamtlichen Partizipation einhergeht, beispielsweise wenn beim Verlassen der Kinder des elterlichen Haushaltes Gelegenheiten, sich als Elternvertretung oder im Sportverein der Kinder zu engagieren, wegfallen. Vor allem für Mütter, die vergleichsweise häufig im Kontext von Kindergarten und Schu- le ehrenamtlich tätig sind, könnten die Möglichkeiten, sich ehrenamtlich zu engagieren, somit mit steigendem Alter weniger werden. Umgekehrt könnte aber auch besonders in der Familienphase eine höhere Belastung von Frauen zu erwarten sein, welche die ehrenamtliche Partizipation beschränken könnte - ähnlich wie die Erwerbspartizipation. Da Frauen noch immer häufiger und im größeren zeitlichen Umfang Sorgetätigkeiten in der Familie übernehmen, sind sie somit durch die Übernahme von familiären Verpflichtungen stärker eingeschränkt als Männer (vgl. Kapitel 6 Unbezahlte Sorgetätigkeiten in diesem Band). Bei Männern könnten sich hingegen zusätzlich Gelegenheiten zum Engagement über andere Lebensbereiche wie der beruflichen Tätigkeiten in allen biografischen Phasen bis in den Ruhestand hinein ergeben (zur Bedeutung der früheren beruflichen Tätigkeit für das Engagement im Alter vgl. Maurer 2018).

Untersucht wird, ob sich das ehrenamtliche Engagement im Alternsverlauf von Frauen und Männern unterscheidet. Erwerbstätigkeit und familiäre Verpflichtungen beeinflussen das ehrenamtliche Engagement, so dass die ehrenamtliche Partizipation sich mit dem Alter und den verschiedenen Lebensphasen entwickelt. Da die Familienphase, etwa Kindererziehungszeiten, aber auch Sorgetätigkeiten wie Pflege, die Erwerbspartizipation der Frauen in jüngerem Alter anders als die der Männer beeinflusst, ist zu erwarten, dass das ehrenamtliche Engagement der Frauen auch in der Familienphase anders ist als das der Männer. Oder allgemeiner ausgedrückt, dass das Engagement von Frauen und Männern sich im Verlauf zwischen 40 und 90 Jahren unterscheidet. Mit den im nachfolgenden präsentierten Längsschnittanalysen lässt sich erstmals belegen, wie stark sich die Wahrscheinlichkeit, sich ehrenamtlich zu engagieren, für Frauen und Männer mit dem Älterwerden verändert. 


\section{Sozialer Wandel der Alternsverläufe ehrenamtlichen Engagements}

Das ehrenamtliche Engagement hat sich in den letzten 20 Jahren stark ausgeweitet, der Anteil Engagierter hat sich nahezu verdoppelt: 1996 waren rund 12 Prozent der 40- bis 85-Jährigen ehrenamtlich in Vereinen oder Organisationen engagiert, 2014 waren es bereits gut 22 Prozent (Wetzel \& Simonson 2017). Diesen Anstieg gab es sowohl bei den Frauen (von 8 Prozent auf 19 Prozent) als auch bei den Männern (von 17 Prozent auf 26 Prozent). Dieser Trend ist auch durch Befunde anderer Studien wie dem Deutschen Freiwilligensurvey zu belegen, in dem auch freiwilliges Engagement berücksichtigt wird, welches nicht organisationsgebunden ist (vgl. Vogel et al. 2017). Die auf Basis des Deutschen Alterssurveys ausgewiesenen Quoten ehrenamtlichen Engagements beschreiben organisationsgebundenes ehrenamtliches Engagement. Der Anstieg des Engagements ist laut Deutschem Alterssurvey besonders bei jenen über 65 Jahren überdurchschnittlich ausgeprägt (vgl. Wetzel \& Simonson 2017: 83). Es kann daher vermutet werden, dass sich die Möglichkeiten, sich ehrenamtlich zu engagieren, für später geborene $\mathrm{Ge}-$ burtsjahrgänge verbessert haben. Eine Analyse auf Basis des Sozio-oekonomischen Panels (SOEP) hat ergeben, dass der Anstieg des Engagements zum Teil auf einen Kohortenwandel zurückzuführen ist, denn die vor 1935 Geborenen haben sich seltener ehrenamtlich engagiert als die 1935 bis 1945 geborene „Adenauer-Generation“ (Şaka 2018: 288). Die später geborenen Kohorten der „APO-Generation“ (1946 bis 1953 geboren) und der „Generation der Neuen Sozialen Bewegungen“ (1954 bis 1964 geboren) zeigen jedoch nach Kontrolle von Einflussgrößen wie Bildung keine zusätzliche höhere Engagementbeteiligung (Şaka 2018: 289).

Der Anstieg lässt sich vermutlich unter anderem darauf zurückführen, dass die verstärkte Engagementförderung durch die Politik Früchte trägt. Hierzu zählen neben der Förderung von Anlaufstellen für Engagementinteressierte auch die Verbesserung der Anerkennungskultur durch Prei- se und Auszeichnungen oder Ähnliches. Auch die Rahmenbedingungen für Engagement, wie die Klärung des Versicherungsschutzes, haben sich verbessert (vgl. Wetzel \& Simonson 2017: 83). Neben diesen Verbesserungen auf politischer und gesellschaftlicher Ebene gibt es Gründe für den Engagementanstieg auf der individuellen und auf der organisationalen Ebene: Auf der individuellen Ebene ist zu nennen, dass Personen, die heute älter sind, besser ausgebildet sind als dies die Älteren von früher waren. Da Bildung eine wichtige Ressource für ehrenamtliches Engagement ist, lässt sich der Anstieg des Engagements bei den älteren Menschen zum Teil auf die Ausweitung der Qualifikationen zurückführen. Insbesondere sind Frauen heute zu deutlich höheren Anteilen hochgebildet als in der Vergangenheit, weshalb sich eine stärkere Zunahme der Ehrenamtsbeteiligung bei älteren Frauen erwarten lässt (vgl. Vogel et al. 2017: 118; Şaka 2018). Gleichzeitig ist die Erwerbsbeteiligung in den letzten Jahren deutlich angestiegen - vor allem bei Frauen - und das Ruhestandeintrittsalter verschiebt sich weiter nach hinten. Da Erwerbsarbeit Gelegenheiten zum Engagement mit sich bringt, kann sich auch dieser Trend als engagementförderlich erweisen (vgl. Wetzel \& Simonson 2017: 83). Darüber hinaus trägt auch eine bessere Gesundheit dazu bei, dass Menschen sich vermehrt bis ins hohe Alter ehrenamtlich engagieren können. Auf der individuellen Ebene ist die Situation der Familien zu nennen: Es zeigen sich Veränderungen in den Familienstrukturen, zum Beispiel gibt es zunehmend mehr Personen ohne eigene Kinder. Möglicherweise werden in Zukunft wegfallende familiale Hilfestrukturen mehr durch ehrenamtliche Hilfestrukturen ersetzt (vgl. Vogel et al. 2017: 118). Auf der organisationalen Ebene sind als weitere Gründe für den Anstieg des Anteils ehrenamtlich engagierter älterer Menschen zu nennen, dass die Zahl der Vereine in Deutschland stetig steigt und damit die Nachfrage nach Personen, die ehrenamtlich Vereinsfunktionen wie Kassenwart und Vorsitz etc. übernehmen (vgl. Vogel et al. 2017: 118). Insbesondere sind hier auch Vereinsgründungen von Bürgerhilfevereinen $\mathrm{zu}$ 
nennen, deren Zweck häufig in der Hilfe für ältere Menschen und der Unterstützung bei einem selbstständigen Leben bis ins hohe Alter besteht (vgl. z. B. Alisch, Ritter, Glaser, \& Rubin 2018).

Es ist eine offene Frage, ob sich die Geschlechterunterschiede beim ehrenamtlichen Engagement im Alternsverlauf bei später geborenen Kohorten im Vergleich zu früher geborenen Kohorten weniger ausgeprägt darstellen. Eine Reduzierung der Unterschiede im ehrenamtlichen Engagement von älteren Frauen und Männern könnte beispielsweise erwartet werden, weil Frauen stärker von der Bildungsexpansion profitieren konnten als Männer und Frauen der jüngeren Kohorten eine höhere Erwerbspartizipation ausüben als Frauen der älteren Kohorten. Es liegen Studien vor, die zeigen, dass es eine positive Beziehung zwischen Erwerbstätigkeit und außerberuflicher Partizipation gibt (Künemund \& Schupp 2008; Naumann \& Romeu Gordo 2010; Simonson \& Hameister 2017). Wenn Personen nicht mehr erwerbstätig sind, beenden sie auch eher ihr Ehrenamt. Neben einer Verringerung der Geschlechterunterschiede im Alternsverlauf ist aber auch eine Verstärkung zu erwarten, weil die intensivere Erwerbstätigkeits- und Familienphase zwischen 40 und 60 einen Verdrängungseffekt auf die außerberufliche gesellschaftliche Partizipation insbesondere von Frauen später geborener Kohor- ten haben kann. Nach dem Erwerbsaustritt würde dieser Verdrängungseffekt wegfallen.

\section{Fragestellungen}

Wir analysieren in diesem Kapitel die kohortenspezifischen Alternsverläufe von Frauen und von Männern mit Blick auf ihr ehrenamtliches Engagement. Dabei erlaubt die Datenstruktur des Deutschen Alterssurveys, die früher geborene Kohorte der 1930 bis 1939 Geborenen mit den später geborenen Kohorten der Geburtsjahrgänge 1940 bis 1949 und 1950 bis 1959 zu vergleichen. Während die in den 1930er Jahren Geborenen heute bereits ein hohes Alter erreicht haben, hat ein Großteil der in den 1950er Jahren Geborenen heute bereits den Übergang in den Ruhestand erfahren oder steht kurz vor dem Übergang in den Ruhestand. Im Vordergrund stehen die Fragen, wie sich das ehrenamtliche Engagement später geborener Kohorten über die zweite Lebenshälfte hinweg unterscheidet vom ehrenamtlichen Engagement früher geborener Kohorten, und ob sich die Geschlechterunterschiede im ehrenamtlichen Engagement in den Alternsverläufen später geborener Kohorten verringern.

\section{Indikatoren}

Organisationsgebundenes ehrenamtliches Engagement wird im Deutschen Alterssurvey bei Personen erfasst, die Mitglied in einer Gruppe oder einer Organisation sind, z.B. in einem Sportverein, in einer religiösen Gruppe oder in der Freiwilligen Feuerwehr. Als ehrenamtlich engagiert geht in die Analysen ein, wer angibt, Mitglied in einer Gruppe oder Organisation zu sein und im Anschluss die Frage bejaht „Üben Sie dort eine Funktion aus oder haben Sie ein Ehrenamt inne?“. Da 
viele Menschen in mehreren Vereinen oder Organisationen tätig sind, werden maximal fünf Mitgliedschaften pro Person erfasst. Die im Interview verwendete Liste der Gruppen und Organisationen umfasst die Kategorien: Selbsthilfegruppen, Bürgerinitiativen, Wohltätige Organisationen, die Freiwillige Feuerwehr, Hobby- und Sammelvereine, Gesellige Vereinigungen, Sportvereine, Flüchtlings- oder Vertriebenenverbände, Gewerkschaften, Unternehmens- und Berufsverbände, Politische Parteien, Kirchliche oder religiöse Gruppen, Heimatvereine oder Bürgervereine, Kulturvereine, Musik- und Tanzgruppen sowie Andere.

Personen ab 50 Jahren werden zusätzlich gefragt, ob sie in einer Gruppe mitmachen, die sich besonders „an ältere Menschen im Ruhestand oder im Übergang in den Ruhestand richten“. Die im Interview verwendete Liste umfasst diese Kategorien: Vorruhestandsgruppen oder Gruppen zur Vorbereitung auf den Ruhestand, Seniorenselbsthilfegruppen oder Seniorengenossenschaften, Gruppen für freiwillige Tätigkeiten und Hilfen, Seniorenarbeit der Parteien oder Gewerkschaften sowie Seniorenvertretung und -beiräte, Seniorenuniversitäten und -akademien oder Weiterbildungsgruppen, betriebliche Seniorengruppen, Seniorenfreizeitstätten oder Seniorentreffpunkte, andere Seniorengruppen. Als ehrenamtlich engagiert geht in die Analysen ein, wer bezüglich einer solchen Gruppe die Frage bejaht „Üben Sie dort eine Funktion aus oder haben Sie ein Ehrenamt inne?". Auch hier können Personen wieder bis zu fünf Gruppen nennen.

Um Aussagen über ehrenamtliches Engagement insgesamt treffen zu können, wird zusammen betrachtet, ob sich Menschen allgemein in Organisationen oder aber in Seniorenorganisationen engagieren. Da ehrenamtliches Engagement darüber hinaus auch außerhalb von Mitgliederorganisationen wie Vereinen oder Verbänden ausgeübt werden kann, etwa im kommunalen Bereich als Schöf$\mathrm{fe}$, als ehrenamtlicher Bürgermeister oder in der informellen Nachbarschaftshilfe, ist für die Interpretation der Ergebnisse dieses Kapitels von Bedeutung, dass es sich allein um organisationsge- bundenes ehrenamtliches Engagement und somit nur um einen Ausschnitt des von Menschen in der zweiten Lebenshälfte ausgeübten ehrenamtlichen Engagements insgesamt handelt. Die Anteile ehrenamtlich Engagierter ist im Deutschen Alterssurvey möglicherweise minimal überschätzt, da Menschen, die sich ehrenamtlich engagieren, auch häufiger bereit sind an wissenschaftlichen Studien teilzunehmen, als Menschen, die sich nicht engagieren (vgl. u. a. Abraham, Helms und Presser 2009). Dennoch lassen sich hier gültige Aussagen über die Wahrscheinlichkeit ehrenamtlichen Engagements im Alternsverlauf im Sinne von mit dem Alter steigenden oder sinkenden Wahrscheinlichkeiten treffen.

In der Darstellung der Ergebnisse unterscheiden wir zwischen der mittleren Wahrscheinlichkeit, ein Ehrenamt auszuüben, die im Folgenden als "Ausübung eines Ehrenamtes" beschrieben wird, der mittleren Wahrscheinlichkeit, ein Ehrenamt aufzunehmen und der mittleren Wahrscheinlichkeit, ein Ehrenamt zu beenden, die als „Neuaufnahme eines Ehrenamtes“ und als „Beendigung eines Ehrenamtes" beschrieben werden. Personen erhalten den Wert 1 für „Neuaufnahme“, wenn sie in der betrachten Erhebungswelle ein Ehrenamt ausüben, in der vorherigen Erhebungswelle aber kein Ehrenamt ausgeübt haben. Den Wert 0 "Keine Neuaufnahme“ erhalten alle Personen, die in der betrachteten Erhebungswelle kein Ehrenamt ausüben sowie Personen, die in der betrachteten Erhebungswelle ein Ehrenamt ausüben, aber auch in der vorherigen Erhebungswelle bereits ein Ehrenamt ausgeübt haben. Das Vorgehen für die Beendigung ist äquivalent (Wert 1 „Beendigung“ erhalten Personen, die in der betrachteten Erhebungswelle kein Ehrenamt ausüben, in der vorherigen Erhebungswelle aber ein Ehrenamt ausgeübt haben).

\section{Statistisches Analyseverfahren}

Untersucht wird, wie hoch die durchschnittliche Wahrscheinlichkeit in der Population über die Al- 
tersspanne von 40 bis 90 Jahren ist, zu einem bestimmten Alter im Alternsverlauf eine ehrenamtliche Tätigkeit auszuüben. Zudem wird untersucht, wie hoch die durchschnittliche Wahrscheinlichkeit im Alter von 40 bis 90 Jahren ist, zu einem bestimmten Alter eine neue ehrenamtliche Tätigkeit aufzunehmen beziehungsweise im Alternsverlauf eine ehrenamtliche Tätigkeit zu beenden. In der Ergebnisdarstellung werden die in Analysemodellen geschätzten Wahrscheinlichkeiten als Prozentanteile ausgegeben. Auf Basis des Designs des Deutschen Alterssurveys ist es möglich, den gesamten Verlauf über 50 Jahre (also von 40 bis 90 Jahre) zu schätzen. Für jeden Geburtsjahrgang liegen Angaben über einen Zeitraum von maximal 21 Jahren zugrunde (zum Stichprobendesign siehe Kapitel 2 Design, Inhalte und Methodik des Deutschen Alterssurveys in diesem Band).

Die Modellschätzungen erfolgten mit Hilfe logistischer Panelregressionen. Es wurden eine li- neare sowie eine quadratische Alterskomponente aufgenommen, um die linearen und kurvilinearen Alternsveränderungen der Wahrscheinlichkeiten, sich zu engagieren, ein Ehrenamt neu aufzunehmen oder ein Ehrenamt zu beenden, abzubilden. Außerdem wurde für Geschlecht und Region kontrolliert, um der geschichteten Stichprobenziehung der DEAS-Basiserhebungen Rechnung zu tragen. Die Unterschiede zwischen den Geburtskohorten wurden anhand der Annahme, dass sich Kohorteneffekte für jede später geborene Geburtskohorte in gleicher Form darstellen werden, als lineare Kohorteneffekte geschätzt (vgl. hierzu ausführlich das Kapitel 2 Design, Inhalte und Methodik des Deutschen Alterssurveys in diesem Band). Die Alternsveränderungen und die Unterschiede nach Geschlecht oder Kohorte wurden in den Modellschätzungen auf statistische Signifikanz getestet, die Irrtumswahrscheinlichkeit wurde bei allen Tests auf $\mathrm{p}<0,05$ festgesetzt.

\subsection{Ergebnisse}

\subsubsection{Ausübung eines Ehrenamtes im Verlauf der zweiten Lebenshälfte}

In Abbildung 7-1 ist der durchschnittliche Alternsverlauf der Ausübung ehrenamtlicher Tätigkeiten separat für Frauen und Männer sowie für die gesamte Untersuchungsstichprobe dargestellt. Allgemein ist die ehrenamtliche Partizipation in Vereinen und Organisationen (nach der Definition von Abschnitt 2) über den hier betrachteten Lebensverlauf von 40 bis 90 Jahren niedrig. In dem analysierten Altersbereich von 40 bis 90 Jahren liegen die Werte unter 20 Prozent für Frauen und knapp über 20 Prozent für Männer. Am höchsten ist die Wahrscheinlichkeit, sich ehrenamtlich $\mathrm{zu}$ engagieren, im Alter zwischen 53 und 63 Jahren.

Über die zweite Lebenshälfte steigt die Wahrscheinlichkeit, sich ehrenamtlich zu engagieren, zunächst an, später sinkt sie mit steigendem Lebensalter jedoch wieder $a b$.

Vom Alter von 40 bis zum Alter von 58 Jahren steigt die Wahrscheinlichkeit stetig an, sich ehrenamtlich zu engagieren (Abbildung 7-1). Ab einem Alter von 59 Jahren nimmt die Wahrscheinlichkeit, ehrenamtlich engagiert $\mathrm{zu}$ sein, mit steigendem Alter stetig ab (Abbildung 7-1).

Über die gesamte zweite Lebenshälfte hinweg haben Männer eine höhere Wahrscheinlichkeit, sich ehrenamtlich zu engagieren, als Frauen.

Bei Männern steigt die durchschnittliche Wahrscheinlichkeit, ehrenamtlich engagiert zu sein, von 17 Prozent (im Alter von 40 Jahren) auf ein Maximum von 23 Prozent (mit 57 Jahren) und sinkt später auf sieben Prozent (mit 90 Jahren). Bei Frauen steigt diese Wahrscheinlichkeit von elf Prozent auf 
Abbildung 7-1 Ausübung eines Ehrenamtes bei Frauen und Männern im Alternsverlauf

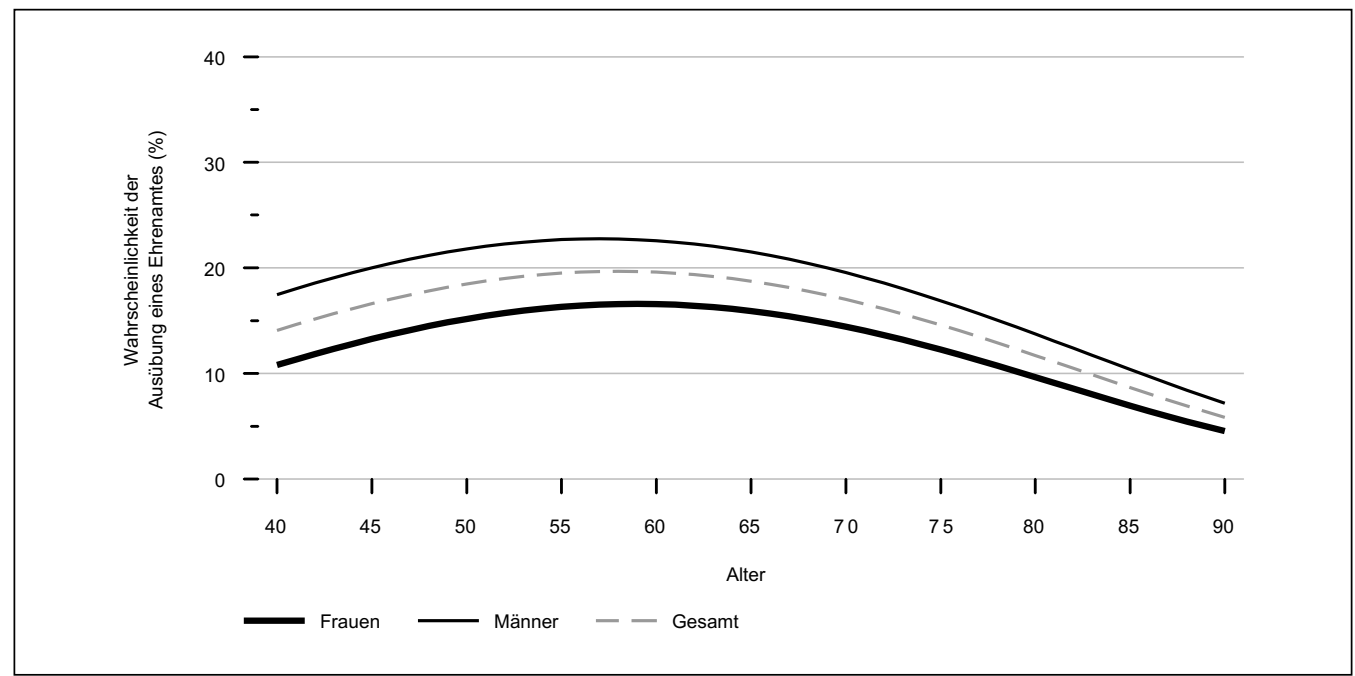

Quelle: DEAS 1996-2017, Schätzungen basieren auf n = 20070 Studienteilnehmenden (10 174 Männer und 9876 Frauen) mit insgesamt $\mathrm{n}=39280$ Beobachtungen; kontrolliert für Region.

Bei Frauen steigt die Wahrscheinlichkeit, ehrenamtlich engagiert zu sein, von elf Prozent (mit 40 Jahren) auf ein Maximum von 17 Prozent (mit 6o Jahren) und sinkt auf fünf Prozent (mit 90 Jahren). Bei Männern steigt die Wahrscheinlichkeit, ehrenamtlich engagiert zu sein, auf ein Maximum von 23 Prozent (mit 6o Jahren) und sinkt später auf sieben Prozent ab (mit 90 Jahren).

ein Maximum von 17 Prozent (mit 59 Jahren) und sinkt mit steigendem Lebensalter auf fünf Prozent (mit 90 Jahren). Über den gesamten Alternsverlauf ist die Wahrscheinlichkeit der Ausübung eines Ehrenamtes somit höher für Männer als für Frauen (Abbildung 7-1). Allerdings sind die Geschlechterunterschiede der Wahrscheinlichkeit, ein Ehrenamt auszuüben, in der Erwerbsphase größer als in der Ruhestandsphase (ab einem Alter von 88 Jahren ist der Unterschied zwischen Frauen und Männern nicht mehr statistisch signifikant).

Die Wahrscheinlichkeit, ehrenamtlich engagiert zu sein, ist für später geborene Kohorten über den gesamten betrachteten Alternsverlauf höher als für früher geborene Kohorten.

In Abbildung 7-2 sind die Alternsverläufe für drei Geburtskohorten dargestellt: für die zwischen 1930 und 1939 Geborenen; für die zwischen 1940 und
1949 Geborenen und für die zwischen 1950 und 1959 Geborenen. Allgemein kann festgehalten werden, dass später geborene Kohorten eine höhere Wahrscheinlichkeit ehrenamtlicher Partizipation im Alternsverlauf zeigen als früher geborene Kohorten (Abbildung 7-2). Die Form der Wahrscheinlichkeitsverläufe scheint für die Kohorten ähnlich, für alle drei Geburtskohorten steigt die Wahrscheinlichkeit, ehrenamtlich tätig zu sein, zunächst mit dem Lebensalter an. Darüber hinaus scheint sich der Wendepunkt, zu dem ein Rückgang des Ehrenamtes mit steigendem Alter eintritt, biografisch nach hinten zu verschieben. Während für die früher geborene Kohorte (1930 bis 1939 geboren) ein Rückgang ab einem Alter von 70 Jahren zu beobachten ist, ist das nicht der Fall für die zwischen 1940 und 1949 geborenen Personen. Für diese mittlere Kohorte beobachten wir die Partizipation bis 77 Jahre, jedoch ist bis zu diesem Alter kein Rückgang zu beobachten, der Wendepunkt 
tritt also voraussichtlich biografisch noch später ein. Auch der Verlauf der jüngsten Kohorte (1950 bis 1959) zeigt in dem zuletzt beobachteten Alter (67 Jahre) immer noch eine steigende Tendenz. Das heißt, die Wahrscheinlichkeit, im höheren Alter engagiert zu sein, ist bei den später Geborenen höher als bei den früher Geborenen, sie steigt mit dem Alter über eine ausgedehntere Altersspanne an und sie nimmt voraussichtlich erst ab einem höheren Alter ab.

Wenn sich die derzeitigen gesellschaftlichen Bedingungen in der Zukunft nicht gravierend verändern, werden sich somit voraussichtlich immer mehr Personen auch in einem höheren Lebensalter ehrenamtlich engagieren. Unsere Analysen zeigen, dass sich der Wendepunkt, zu dem ein Rückgang der Wahrscheinlichkeit, ein Ehrenamt auszuüben, erfolgt, in der zweiten Lebenshälfte weiter nach hinten verschieben wird. Voraussichtlich wird sich die Phase, in der Personen im Alter ehrenamtlich aktiv bleiben, somit verlängern.
Die Geschlechterunterschiede im Alternsverlauf reduzieren sich nicht für später geborene Kohorten.

Sowohl für Frauen als auch für Männer ist die Wahrscheinlichkeit, ehrenamtlich engagiert $\mathrm{zu}$ sein, für später geborene Kohorten über den gesamten beobachteten Alternsverlauf höher als für früher geborene Kohorten. Abbildung 7-3 zeigt für alle drei Kohorten, dass die Wahrscheinlichkeit, ein Ehrenamt auszuüben, für Männer höher ist als für Frauen und das zu jedem Zeitpunkt im Alternsverlauf. Der in Abbildung 7-2 dargestellte soziale Wandel im Ehrenamt unterscheidet sich nicht für Frauen und Männer. Das bedeutet auch, dass die Geschlechterunterschiede im Alternsverlauf in jeder Kohorte relativ konstant bleiben. Dass sich die Ehrenamtsbeteiligungen der Frauen und Männer im Alternsverlauf über die Kohorten ähnlich entwickeln, bedeutet also, dass es keine Annäherung der Ehrenamtswahrscheinlichkeit zwischen den Geschlechtern gibt.

\section{Abbildung 7-2 Ausübung eines Ehrenamtes nach Kohorten im Alternsverlauf}

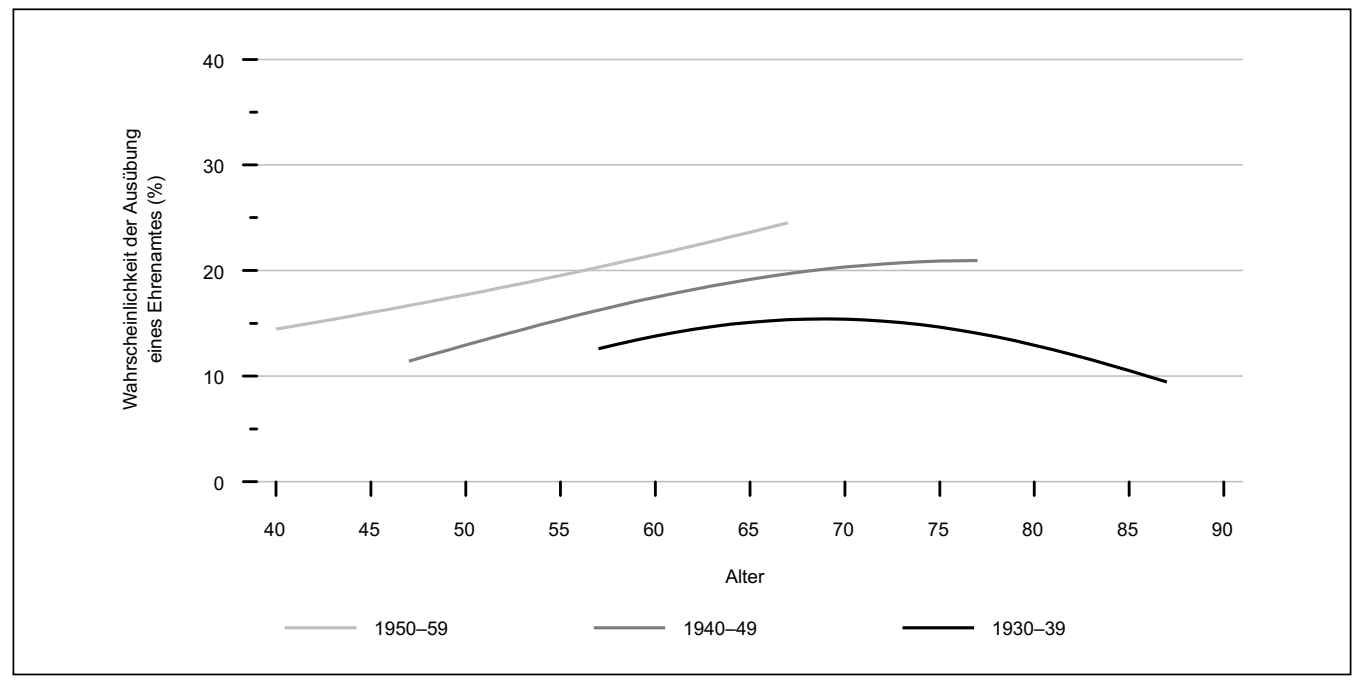

Quelle: DEAS 1996-2017, Schätzungen basieren auf n = 13966 Studienteilnehmenden mit insgesamt n = 29125 Beobachtungen; kontrolliert für Region und Geschlecht.

Für die älteste Kohorte (1930 bis 1939) ist ein Rückgang im ehrenamtlichen Engagement ab einem Alter von 72 Jahren zu beobachten. Für die zwischen 1940 und 1949 geborenen Personen ist bis zum Alter von 77 Jahren (bis zu diesem Alter ist diese Kohorte im DEAS zu beobachten) kein Rückgang zu beobachten. 
Abbildung 7-3 Ausübung eines Ehrenamtes bei Frauen und Männern nach Kohorten im Alternsverlauf

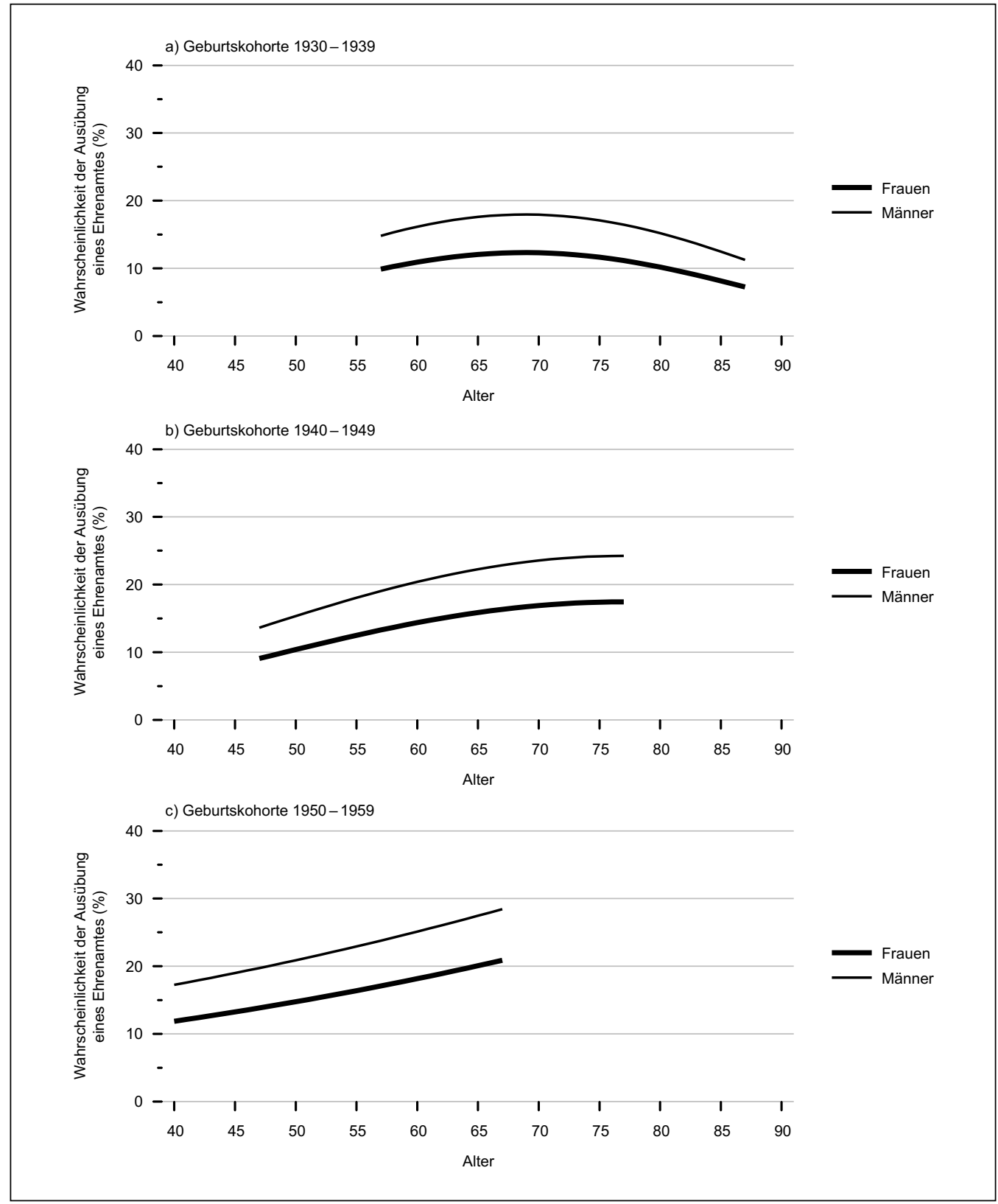

Quelle: DEAS 1996-2017, Schätzungen basieren auf $n=13966$ Studienteilnehmenden mit insgesamt $n=29125$ Beobachtungen; kontrolliert für Region.

Für alle drei Kohorten sind die Alternsverläufe der Wahrscheinlichkeit, ein Ehrenamt auszuüben, für Frauen und Männer ähnlich, so dass es keine Annäherung zwischen den Geschlechtern über die Kohorten hinweg gibt. 


\subsubsection{Neuaufnahme und Beendigung von ehrenamtlichem Engagement im Verlauf der zweiten Lebenshälfte}

Im Durchschnitt steigt die mittlere Wahrscheinlichkeit ehrenamtlicher Partizipation ab 40 Jahren bis ins 6. Lebensjahrzehnt stetig an. Diese Steigerung kommt dadurch zustande, dass in dieser Lebensphase der Anteil von Personen, die ein Ehrenamt neu aufnehmen, höher ist als der Anteil der Personen, die ein solches beenden (Abbildung 7-4).

Die durchschnittliche Wahrscheinlichkeit, ein Ehrenamt auszuüben, ergibt sich zum einen durch die Neuaufnahmen ehrenamtlicher Tätigkeiten und zum anderen durch die Beendigungen ehrenamtlicher Tätigkeiten.

Neuaufnahmen nehmen mit steigendem Lebensalter zunächst bis zu einem Alter von 54 Jahren zu und dann nehmen sie mit steigendem Lebensalter ab. Das heißt, mit höherem Lebensalter werden die Personen, die zuvor nicht ehrenamtlich tätig waren, auch seltener ehrenamtlich aktiv. Es sollte beachtet werden, dass auch wenn nach dem Erreichen der Regelaltersgrenze des Renteneintritts, die derzeit schrittweise von 65 auf 67 Jahre angehoben wird, immer noch Personen ein neues Ehrenamt aufnehmen, die Neuaufnahmen in diesem Alter somit niedriger sind als vor dem Renteneintritt. Die Personen fangen eher mit einem Ehrenamt an, wenn sie im Erwerbsalter sind als wenn sie sich bereits im Ruhestandsalter befinden (Abbildung 7-4). Darüber hinaus ist bis zu einem Alter von 64 Jahren die Wahrscheinlichkeit von Personen, die eine ehrenamtliche Aktivität neu aufnehmen, höher als jene von Personen, die eine ehrenamtliche Aktivität beenden. $\mathrm{Ab}$ einem Alter von 65 Jahren bleibt der Anteil der Beendigungen stabil, während die Neuaufnahmen zurückgehen. Die stetig sinkende Wahrscheinlichkeit, im höheren Alter einer ehrenamtlichen Tätigkeit nachzugehen, ergibt sich so-

Abbildung 7-4 Neuaufnahme und Beendigung eines Ehrenamtes im Alternsverlauf

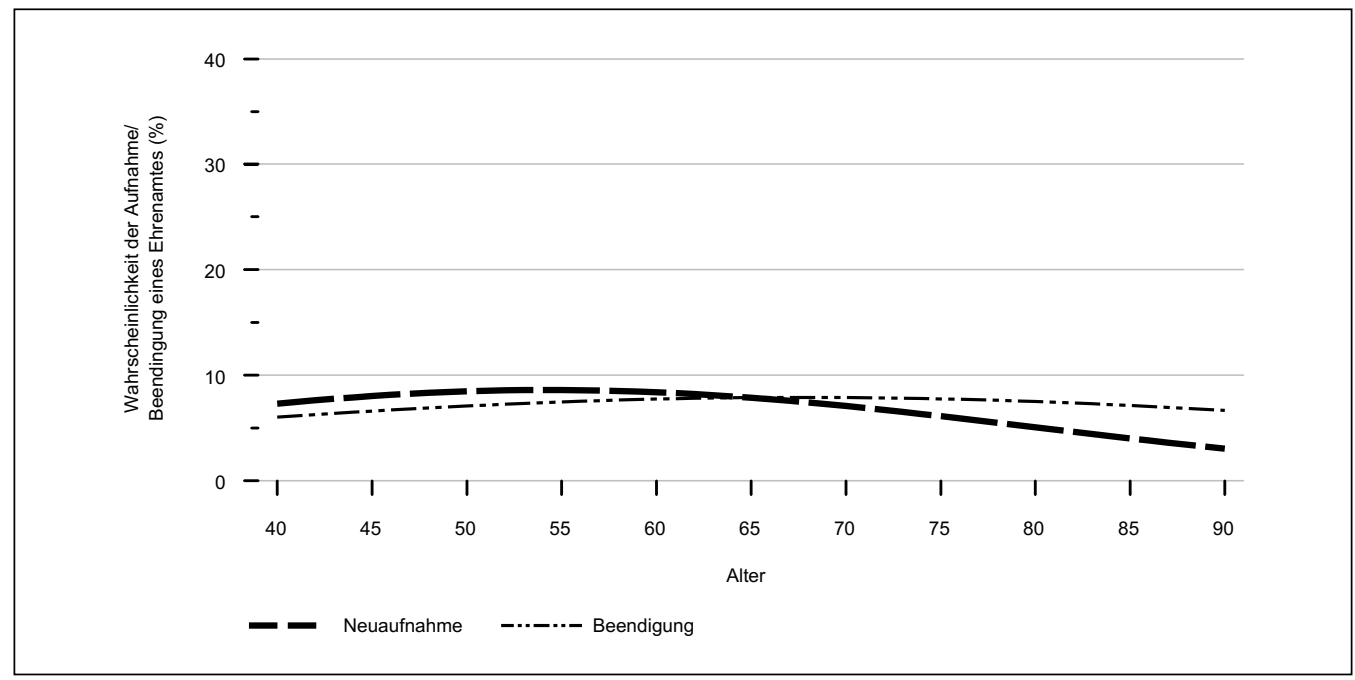

Quelle: DEAS 1996-2017, Schätzungen basieren auf $n=9513$ (Neuaufnahme) und $n=9519$ (Beendigung) Studienteilnehmenden mit insgesamt $\mathrm{n}=18555$ (Neuaufnahme) und $\mathrm{n}=18561$ (Beendigung) Beobachtungen; kontrolliert für Region und Geschlecht.

Die Wahrscheinlichkeit, ein Ehrenamt aufzunehmen, nimmt mit steigendem Lebensalter zunächst bis zu einem Alter von 55 Jahren zu und dann (ab 56 Jahren) mit steigendem Lebensalter ab. Die Wahrscheinlichkeit, ein Ehrenamt zu beenden, verändert sich kaum über den Verlauf der zweiten Lebenshälfte. 
mit daraus, dass in diesem höheren Lebensalter zunehmend weniger Personen ein Ehrenamt neu anfangen und gleichzeitig kaum weniger Engagierte ihre ehrenamtlichen Tätigkeiten beenden. Diese beiden verschiedenen Prozesse (mehr Neuaufnahmen als Beendigungen in der späten Erwerbsphase und weniger Neuaufnahmen als Beendigungen in der Ruhestandsphase) ergeben zusammen das Profil in Abbildung 7-1.

Diese Verläufe bei den Neuaufnahmen und Beendigungen sind sowohl für Frauen als auch für Männer zu beobachten. Bei den Neuaufnahmen sind keine signifikanten Geschlechterunterschiede im Alternsverlauf zu beobachten (Abbildung 7-5). Im Fall der Beendigungsverläufe können nur kleine Unterschiede festgestellt werden (Abbildung 7-6). Während der Verlauf der Frauen stabiler ist und die Wahrscheinlichkeit, ein Ehrenamt zu beenden, nicht die 7-Prozentgrenze übersteigt, liegt bei den Männern der Wendepunkt höher (mit einer Wahrscheinlichkeit von knapp 9 Prozent). Im Alter 40 und im Alter von 90 Jahren sind die Wahrscheinlichkeiten für Frauen und Männer ähnlich.

Die Wahrscheinlichkeit, ein Ehrenamt neu aufzunehmen beziehungsweise zu beenden, ist für später geborene Kohorten über den gesamten betrachteten Alternsverlauf nicht höher beziehungsweise niedriger als für früher geborene Kohorten.

Die Wahrscheinlichkeit, ein Ehrenamt neu aufzunehmen, nimmt im Alter zunächst zu und danach wieder ab, so dass im hohen Alter eine sehr niedrige Wahrscheinlichkeit für eine Neuaufnahme zu beobachten ist. Im Kohortenvergleich beobachten wir ähnliche Verläufe (Abbildung 7-7), wobei bei Frauen und Männern der später geborenen Kohorte (1950-1959) eine höhere Wahrscheinlichkeit im jüngeren Alter zu beobachten ist, die im Alternsverlauf wieder sinkt. Die Unterschiede zwischen den Geburtskohorten sind nicht statistisch signifikant.

Abbildung 7-5 Neuaufnahme eines Ehrenamtes bei Frauen und Männern im Alternsverlauf

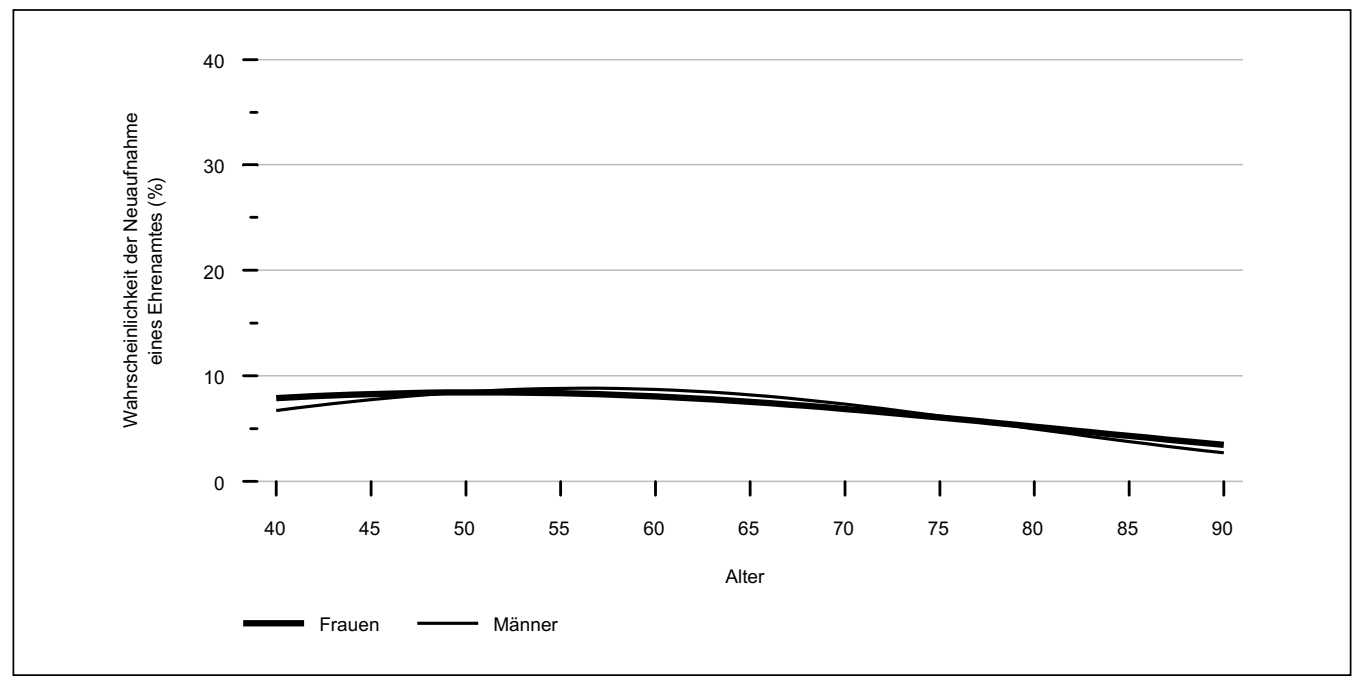

Quelle: DEAS 1996-2017, Schätzungen basieren auf $\mathrm{n}=9513$ Studienteilnehmenden mit insgesamt n = 18555 Beobachtungen; kontrolliert für Region.

Die Wahrscheinlichkeit, ein Ehrenamt neu aufzunehmen, unterscheidet sich nicht signifikant zwischen Frauen und Männern. 


\section{Abbildung 7-6 Beendigung eines Ehrenamtes bei Frauen und Männern im Alternsverlauf}

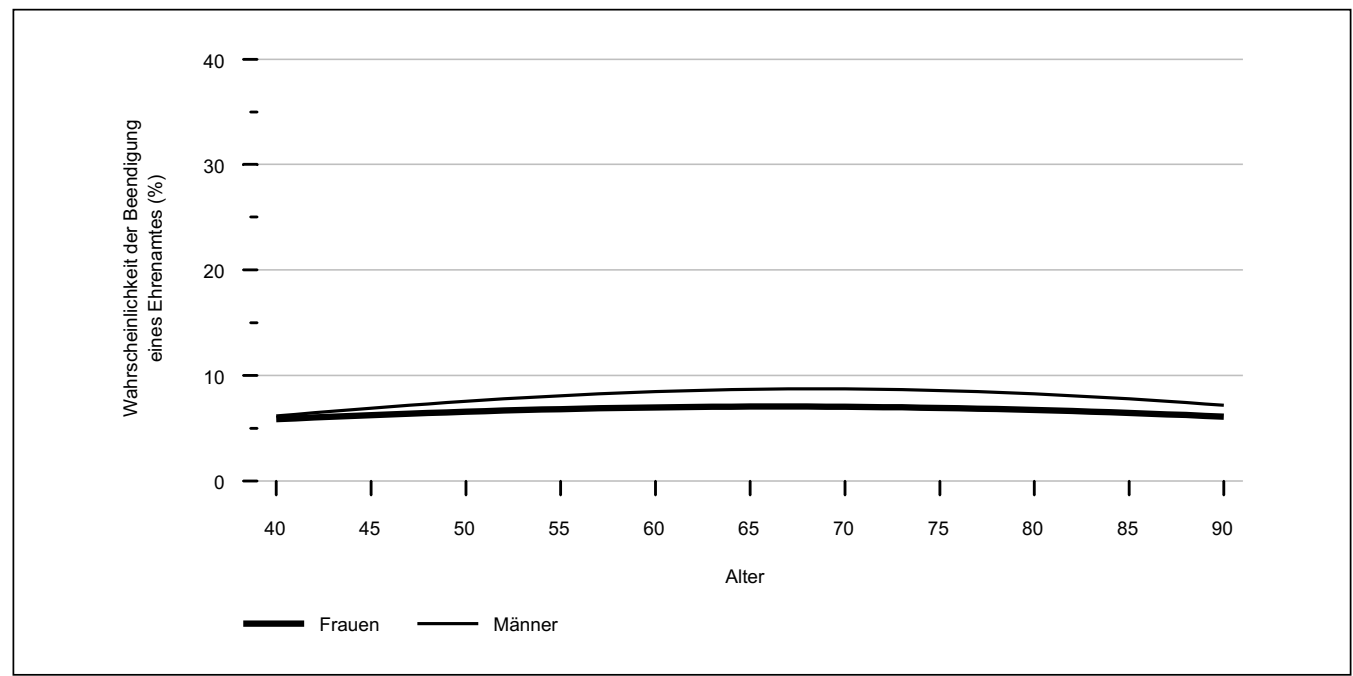

Quelle: DEAS 1996-2017, Schätzungen basieren auf $n=9519$ Studienteilnehmenden mit insgesamt n = 18561 Beobachtungen; kontrolliert für Region.

Die Wahrscheinlichkeit, ein Ehrenamt zu beenden, unterscheidet sich minimal zwischen Frauen und Männern, wobei der Verlauf der Frauen stabiler über den Verlauf der zweiten Lebenshälfte ist.

\section{Abbildung 7-7 Neuaufnahme eines Ehrenamtes nach Kohorten im Alternsverlauf}

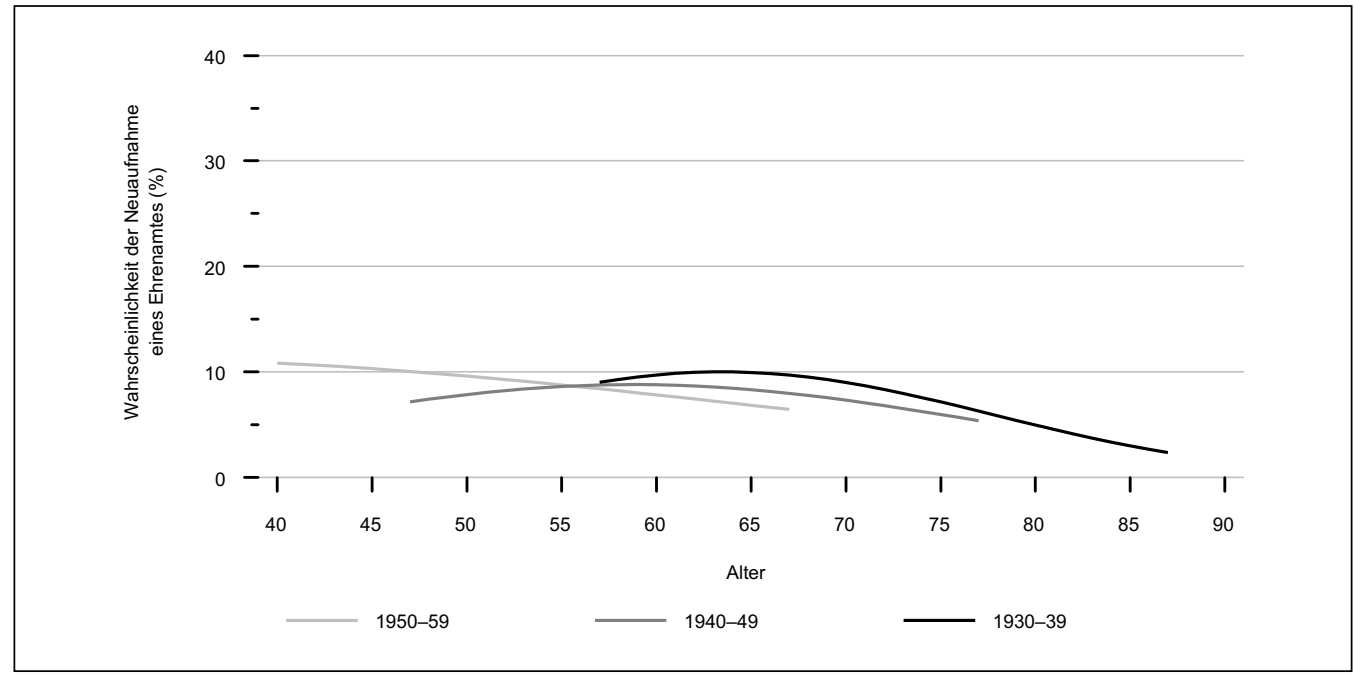

Quelle: DEAS 1996-2017, Schätzungen basieren auf $n=7122$ Studienteilnehmenden mit insgesamt n= 14 625 Beobachtungen; kontrolliert für Region und Geschlecht.

Die Wahrscheinlichkeit, ein Ehrenamt neu anzunehmen, unterscheidet sich im Alternsverlauf nicht signifikant zwischen den Kohorten. 
Abbildung 7-8 Beendigung eines Ehrenamtes nach Kohorten im Alternsverlauf

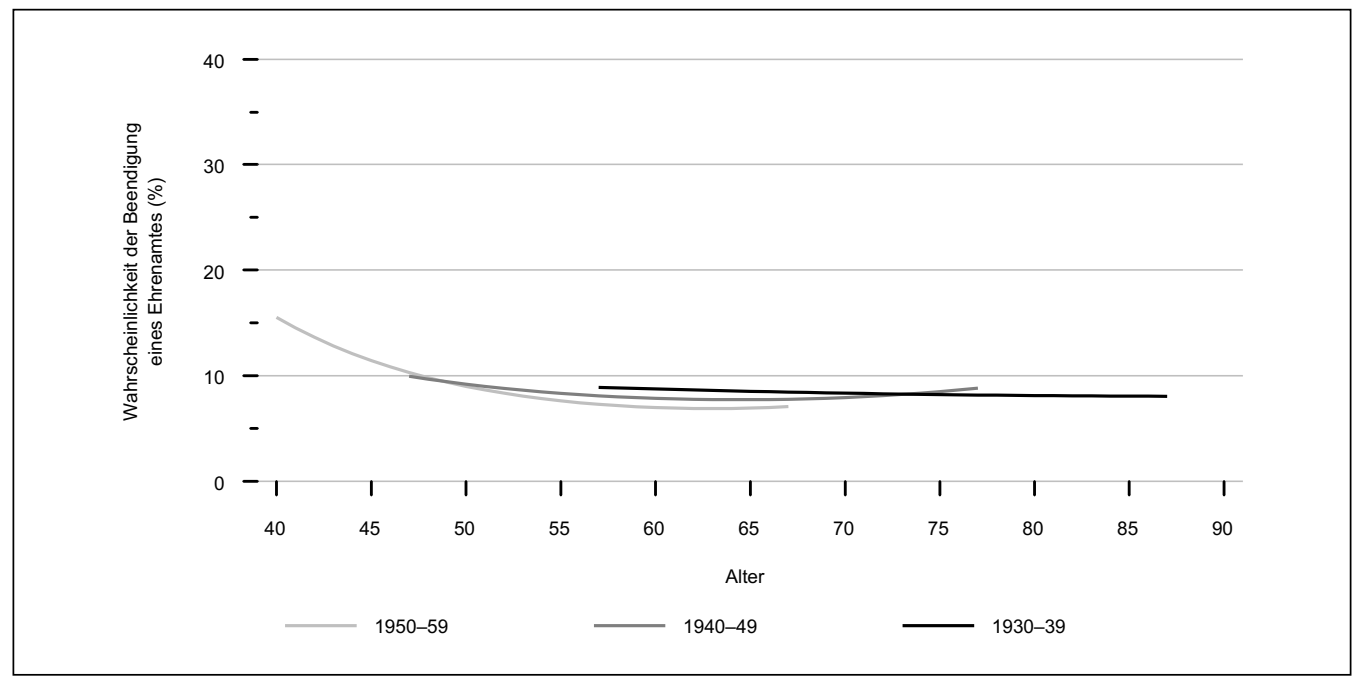

Quelle: DEAS 1996-2017, Schätzungen basieren auf $n=7127$ Studienteilnehmenden mit insgesamt n = 14626 Beobachtungen; kontrolliert für Region und Geschlecht.

Die Wahrscheinlichkeit, ein Ehrenamt zu beenden, unterscheidet sich im Alternsverlauf nicht signifikant zwischen den Kohorten.

Auch bei der Wahrscheinlichkeit, ein Ehrenamt $\mathrm{zu}$ beenden, beobachten wir keinen statistisch signifikanten Unterschied zwischen den Geburtskohorten (Abbildung 7-8). Bei Personen der später geborenen Kohorte (1950-1959) sind höhere Wahrscheinlichkeiten im jüngeren Lebensalter zu beobachten, die dann sinken und sich an die der anderen Geburtskohorten angleichen.

\subsection{Diskussion}

\section{Zusammenfassung}

Sowohl für Frauen als auch für Männer steigt die Wahrscheinlichkeit, sich ehrenamtlich in formalen Organisationen zu engagieren, zunächst mit dem Lebensalter an und sinkt im höheren Alter
Die Geschlechterunterschiede bei der Wahrscheinlichkeit, ein Ehrenamt im Alternsverlauf zu beenden, bleiben für später geborene Kohorten bestehen.

Die geringen Geschlechterunterschiede bei der Wahrscheinlichkeit, ein Ehrenamt zu beenden (Abbildung 7-6), reduzieren sich nicht für später geborene Geburtskohorten. Abbildung 7-9 zeigt, wie diese kleinen Unterschiede zwischen Frauen und Männern relativ konstant bleiben über die Kohorten hinweg.

wieder ab. Der biografische Wendepunkt liegt bei Frauen und Männern bei rund 58 Jahren. Der Geschlechterunterschied besteht hauptsächlich darin, dass Männer über den gesamten betrachteten Verlauf (also von 40 bis 90 Jahren) eine deutlich höhere Wahrscheinlichkeit aufweisen, sich in forma- 
Abbildung 7-9 Beendigung eines Ehrenamtes bei Frauen und Männern nach Kohorten im Alternsverlauf

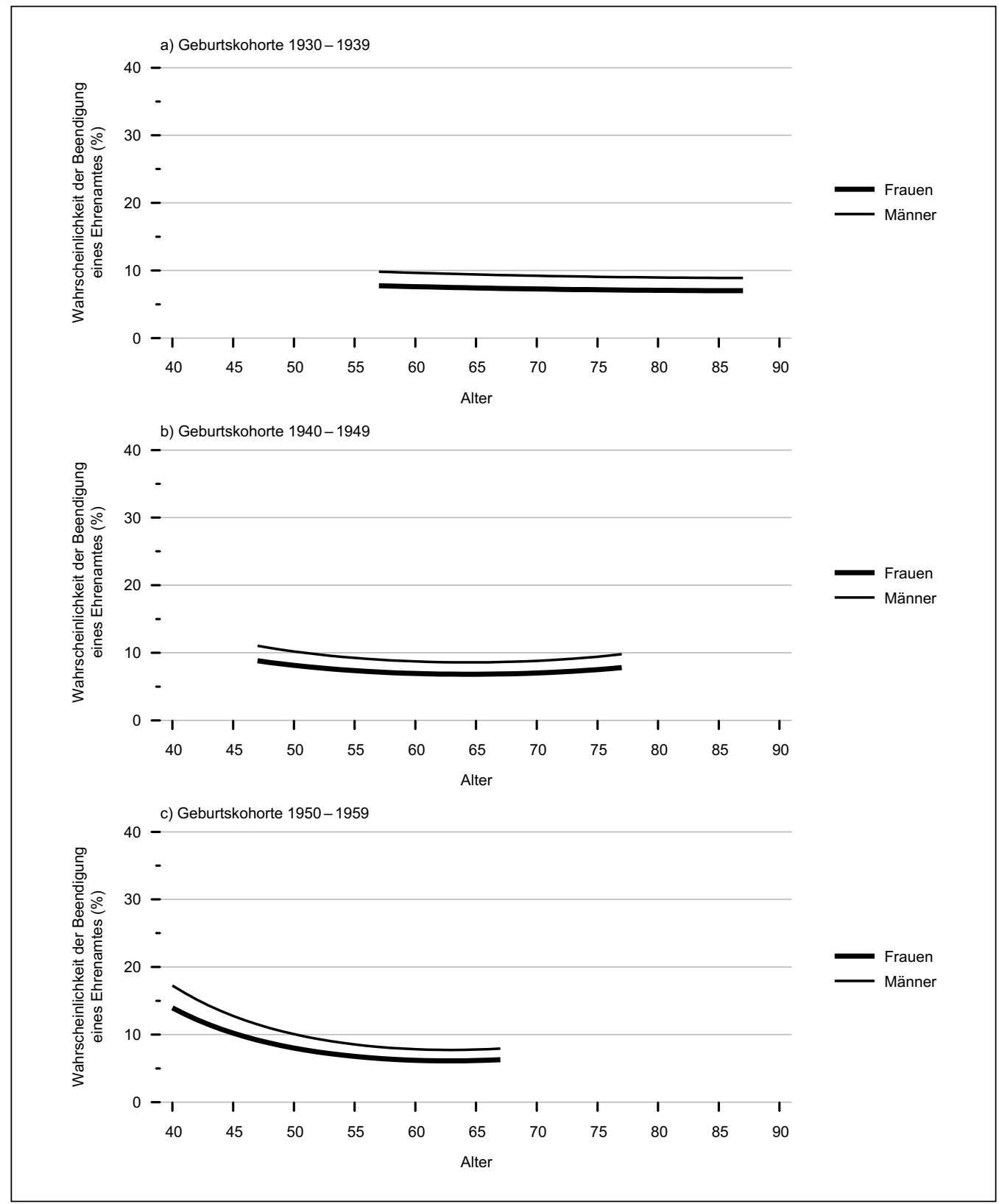

Quelle: DEAS 1996-2017, Schätzungen basieren auf $n=7127$ Studienteilnehmenden mit insgesamt n= 14626 Beobachtungen; kontrolliert für Region.

Die Geschlechterunterschiede in der Wahrscheinlichkeit, im Alternsverlauf ein Ehrenamt zu beenden, bleiben über die Geburtskohorten stabil. 
len Organisationen ehrenamtlich zu engagieren, als Frauen. Dabei ist der Geschlechterunterschied in der Erwerbsphase sogar noch größer als in der Ruhestandsphase, denn der Geschlechterunterschied in der Wahrscheinlichkeit, sich ehrenamtlich zu engagieren, verringert sich mit steigendem Alter insbesondere in der Ruhestandsphase. Später geborene Kohorten haben im Alternsverlauf eine höhere Wahrscheinlichkeit, sich ehrenamtlich zu engagieren, als früher geborene Kohorten. Dieser Befund gilt für Frauen und Männer gleichermaßen. Das bedeutet aber auch, dass die Geschlechterunterschiede in den Alternsverläufen sich über die Kohortenfolge der 1930er, der 1940er und der 1950er Geborenen hinweg nicht verringern.

Die Wahrscheinlichkeit, eine ehrenamtliche Tätigkeit neu aufzunehmen, nimmt bis zu einem Alter von 54 Jahren zu, und sie sinkt dann mit dem Älterwerden ab. Die Beendigung bleibt hingegen im Alternsverlauf stabil. Dadurch ergibt sich die stetig geringere Wahrscheinlichkeit, im höheren Alter einer ehrenamtlichen Tätigkeit nachzugehen. Die Prozesse der Aufnahme von Ehrenämtern unterscheiden sich nicht statistisch signifikant nach Geschlecht und Beendigungsprozesse unterscheiden sich nur minimal nach Geschlecht, mit einer geringfügig höheren Wahrscheinlichkeit bei Männern. Auch im Kohortenvergleich sind keine signifikanten Unterschiede zu erkennen. Die Wahrscheinlichkeit, ein Ehrenamt neu aufzunehmen oder zu beenden, ist für später geborene Kohorten über den gesamten betrachteten Alternsverlauf nicht höher beziehungsweise nicht niedriger als für früher geborene Kohorten.

\section{Interpretation}

Der Anstieg der Wahrscheinlichkeit, ein Ehrenamt auszuüben, zu Beginn der zweiten Lebenshälfte, kann für Frauen und für Männer unter anderem mit Gelegenheiten zum Ehrenamt, die sich durch die berufliche Tätigkeit ergeben, zusammenhängen. Menschen engagieren sich zum Beispiel in Berufsverbänden oder in berufsnahen Tätigkeitsfeldern ehrenamtlich oder der Arbeitgeber ermöglicht eine ehrenamtliche Tätigkeit: Einige Ärztinnen und Ärzte übernehmen etwa ehrenamtlich die Behandlung von Obdachlosen oder Schutzsuchenden, manche Pädagoginnen und Pädagogen kümmern sich um ehrenamtliche Bildungsangebote für Schülerinnen und Schüler mit besonderen Bedarfen. In manchen Betrieben besteht auch die Möglichkeit, sich für Auszubildende mit besonderen Bedarfen etwa durch die Organisation von Lerngruppen oder Trainingsgruppen ehrenamtlich einzusetzen. Der Rückgang der Ehrenamtswahrscheinlichkeit im höheren Alter kann für Frauen und für Männer etwa durch die Beendigung der beruflichen Tätigkeit und dem Wegfall der damit einhergehenden Gelegenheiten zum Ehrenamt, durch gesundheitliche Beeinträchtigungen (siehe Kapitel 3 Funktionale und subjektive Gesundheit in diesem Band) oder durch das Erreichen von - in Gesetzen oder Satzungen festgeschriebenen - Altersgrenzen für ein spezifisches Engagement ebenso erklärt werden, wie durch den Wunsch der Engagierten, keine weiteren Verpflichtungen mehr übernehmen zu müssen.

Wie können die Geschlechterunterschiede bei der Wahrscheinlichkeit, ein Ehrenamt auszuüben, über den Alternsverlauf erklärt werden? Aus der Literatur ist bekannt, dass die Möglichkeiten, sich ehrenamtlich zu engagieren, stark mit Bildung zusammenhängen. Die Geschlechterunterschiede bei der Wahrscheinlichkeit, über den Verlauf der zweiten Lebenshälfte ein Ehrenamt auszuüben, lassen sich zu einem großen Teil auf Bildungsunterschiede zwischen älteren Frauen und Männern zurückführen. ${ }^{1}$ Anders ausgedrückt könnte man sagen, hätten ältere Frauen zu einem ähnlichen Anteil hohe Bildungsabschlüsse wie ältere Männer, soll-

1 Wenn wir in zusätzlichen Analysen die Bildungsniveaus der Männer und Frauen berücksichtigen, sind die Differenzen bei den Ehrenamtswahrschein-

lichkeiten zwischen Frauen und Männern ab einem Alter von 67 Jahren nicht mehr signifikant. 
ten sie sich zu gleichen Anteilen ehrenamtlich engagieren und nicht zu niedrigeren Anteilen.

Wie kann das Weiterbestehen der Geschlechterunterschiede beim ehrenamtlichen Engagement im Alternsverlauf in den nachfolgenden Kohorten erklärt werden? Historisch waren die Bildungschancen für Männer deutlich besser als für Frauen, so dass heute der Anteil von älteren Männern mit hoher Bildung deutlich höher ist als der Anteil von älteren Frauen (Statistisches Bundesamt 2018). Die jüngeren Frauen verfügen heute über ähnliche Bildungsabschlüsse wie jüngere Männer (Statistisches Bundesamt 2018). Obwohl sich in den jüngeren Kohorten der Bildungsunterschied zwischen Frauen und Männern deutlich reduziert hat, zeigen sich jedoch auch bei den jüngeren Kohorten geringere Ehrenamtswahrscheinlichkeiten über den Alternsverlauf bei Frauen als bei Männern. Eine Verringerung der Geschlechterunterschiede ist für das Ehrenamt nicht zu beobachten. Im Kohortenvergleich sind die Entwicklungen für Frauen und Männern ähnlich, so dass jüngere Kohorten für beide Geschlechter eine höhere Partizipation zeigen als früher geborene Kohorten. Dass die Bildungsangleichung nicht zu einer Angleichung von Frauen und Männern beim Ehrenamt führt, liegt unter anderem daran, dass Frauen nach wie vor stärker in familiale Verpflichtungen eingebunden sind als Männer. Diese scheinen sich negativ auf die Übernahme von Ehrenämtern in Organisationen und Vereinen auszuwirken. Die weiterbestehenden Geschlechterunterschiede liegen vermutlich aber unter anderem auch an dem Angebot der zivilgesellschaftlichen Organisationen, das sich mehr an Männer als an Frauen richtet, zum Beispiel gibt es mehr Sportvereine für Männer als für Frauen.

Wie kann der Anstieg der Wahrscheinlichkeit, sich zu engagieren, bei nachfolgenden Kohorten erklärt werden? Dass sowohl Frauen als auch Männer der später geborenen Kohorten eine höhere ehrenamtliche Partizipation im Alternsverlauf zeigen als Frauen und Männer der älteren (also früher geborenen) Kohorten, könnte als Ergebnis der Verbesserung des Gesundheitszustands in der zwei- ten Lebenshälfte (siehe Kapitel 3 Funktionale und subjektive Gesundheit in diesem Band) zu sehen sein. Sowohl Frauen als auch Männer profitieren von einer besseren Gesundheit. Der Geschlechterunterschied in der funktionalen Gesundheit ist allerdings auch nicht kleiner geworden in den später geborenen Kohorten. Also haben Frauen, besonders im höheren Alter, eine schlechtere funktionale Gesundheit als Männer. Das wiederum könnte dazu beitragen, dass die Geschlechterunterschiede in der Wahrscheinlichkeit, sich ehrenamtlich zu engagieren, im höheren Alter stabil geblieben sind. Darüber hinaus können die besseren Engagementchancen später geborener Kohorten auch als Ergebnis der verstärkten Engagementförderung durch die Politik interpretiert werden, allerdings hat diese Engagementförderung bislang nicht $\mathrm{zu}$ mehr Gleichstellung oder einer Verringerung der Geschlechterunterschiede im Ehrenamt geführt.

\section{Politische Implikationen}

Wie soll Engagementförderung in der zweiten Lebenshälfte geschehen? Deutlich geworden ist aus der Analyse der geschlechtsspezifischen Alternsverläufe, dass die Wahrscheinlichkeit, sich in einem bestimmten Alter ehrenamtlich zu engagieren, sowohl getrieben wird durch die Neuaufnahmen ehrenamtlicher Tätigkeiten als auch durch deren Beendigung. Hieraus folgt, dass politische Maßnahmen, die auf eine Verbesserung der Chancen, sich bis ins hohe Alter ehrenamtlich zu engagieren, abzielen, an zwei ganz unterschiedlichen Stellschrauben ansetzen können: 1) An der Verbesserung der Chancen, eine ehrenamtliche Tätigkeit neu aufzunehmen - und zwar insbesondere in einem höheren Lebensalter - bei Personengruppen, die sich bislang eher selten engagieren wie ältere Frauen mit geringer Bildung. 2) An der Verbesserung der Chancen, eine ehrenamtliche Tätigkeit länger auszuüben - auch bis in ein hohes Lebensalter - und nicht zu beenden, für alle Menschen, die sich bereits engagieren und länger engagiert bleiben wollen. Optimal wäre eine am Le- 
bensverlauf orientierte Kombination aus beidem. Menschen, die sich bislang in ihrem Leben noch nicht ehrenamtlich engagiert haben, im höheren Alter dazu in die Lage zu versetzen, wenn sie dies möchten, lenkt den Blick auf die sozial ungleichen Chancen zur gesellschaftlichen Teilhabe durch Ehrenamt etwa von Frauen und Männern.

Wie können die Rahmenbedingungen dafür verbessert werden, dass zum einen mehr Menschen ein Ehrenamt aufnehmen können - wenn sie das wollen - und zum anderen, wie mehr Frauen und Männern dabei unterstützt werden können, ein einmal aufgenommenes Ehrenamt länger im Leben ausüben zu können, und dies möglicherweise auch bis ins hohe Alter hinein? Insbesondere die Gruppe der älteren Arbeitnehmerinnen und Arbeitnehmer, könnte ermutigt werden, durch die Aufnahme eines ehrenamtlichen Engagements auch außerberuflich noch einmal einen Neubeginn zu wagen, der sich vielleicht auch positiv auf die Gestaltung des Übergangs in den Ruhestand auswirken könnte - durch eine Kontinuität im Ehrenamt über den Renteneintritt hinaus. Allerdings ist die Lebensphase um die 60 eine, die insbesondere bei Frauen auch durch private Sorgearbeiten stark belastet ist (vgl. Kapitel 6 Unbezahlte Sorgetätigkeiten in diesem Band). Maßnahmen, die die Neuaufnahme ehrenamtlicher Aufgaben erleichtern sollen, müssen also auch die geschlechterdifferenzierten Belastungen durch berufliche Tätigkeiten wie zum Beispiel in der Familie mit im Blick haben. Hierbei steht weniger die verfügbare Zeit beziehungsweise die aufzuwendende Zeit im Fokus, denn auch Personen, die in Vollzeit erwerbstätig sind, finden zu hohen Anteilen noch Zeit, ein Ehrenamt - das in der Regel nur wenige Stunden pro Woche in Anspruch nimmt - auszuüben. Entscheidend sind vielmehr die Flexibilität der Arrangements einerseits, hier könnten zivilgesellschaftliche Organisationen sicherlich ansetzen und zum Beispiel ihre festen Termine für Vorstandssitzungen etc. flexibilisieren beziehungsweise familienfreundlicher gestalten, und die Belastung durch die Tätigkeiten in verschiedenen Bereichen andererseits. Eine Entlastung für pflegende Angehörige könnte also bei- spielsweise ebenfalls indirekt zu einer höheren Ehrenamtsbeteiligung beitragen. Und wie durch jede Arbeit, können auch im Ehrenamt zeitliche und zum Teil psychische Belastungen durch die jeweilige Tätigkeit entstehen, die vielleicht noch stärker in den Blick der Forschung geraten sollten, um Hindernisse, die dem Engagiertbleiben im Wege stehen, abzubauen.

Maßnahmen, die das längere Engagiertbleiben der Ehrenamtlichen fördern, könnten vor allem für Vereine und Organisationen von Interesse sein, die sich Sorgen machen, genügend Nachwuchskräfte für Leitungs- und Vorstandsfunktionen zu finden. Hier könnten ältere Engagierte mit ihrer Erfahrung abhelfen. Maßnahmen, die darauf abzielen, das Beendigungsalter anzuheben, können indirekt wirken. Zum Beispiel wirken sich Maßnahmen, die zur Aufrechterhaltung der Gesundheit im Alter beitragen, auch positiv auf das Ehrenamt aus. Menschen, denen es gesundheitlich gut geht, können sich auch eher für andere engagieren als Menschen mit gesundheitlichen Einschränkungen. Sie können aber auch direkt wirken, zum Beispiel indem rechtliche und satzungsgemäße Altersgrenzen daraufhin überprüft werden, ob sie verändert, flexibilisiert oder abgeschafft werden können. Eine Abschaffung der Altersgrenzen im Ehrenamt alleine würde jedoch die Geschlechterungleichheit in den Alternsverläufen zementieren, denn jene, die nie begonnen haben, ein Ehrenamt auszuüben (Frauen häufiger als Männer), würde eine solche Maßnahme nicht erreichen. Auch an anderer Stelle sind gruppenunspezifische Maßnahmen möglicherweise sogar kontraproduktiv. Wenn Engagementförderung nach dem Gießkannenprinzip erfolgt, dann werden sich die sozialen Ungleichheiten etwa zwischen den Bildungsgruppen aber eben auch zwischen den Geschlechtern erhalten und reproduzieren, im schlimmsten Falle werden sie sich sogar vergrößern.

Für eine geschlechterdifferenzierende und geschlechtergerechte Engagement- und Altenpolitik ist hierbei von Interesse, ob engagierte Frauen andere Unterstützungsbedarfe haben, um ihr Engagement möglichst lange bis ins hohe Alter auf- 
rechterhalten zu können als Männer und ob an Engagement interessierte Frauen andere Unterstützung benötigen, um ein Ehrenamt aufzunehmen als Männer. Zum Beispiel sollten sich, um das Engagement der Frauen zu erhöhen, beziehungsweise um die Geschlechterunterschiede zu reduzieren, zivilgesellschaftliche Organisationen gezielter an Frauen richten. In den meisten Bereichen haben Frauen geringere Engagementquoten als Männer (vgl. Vogel et al. 2017: 115) und Männer übernehmen auch häufiger Leitungsfunktionen in den Organisationen (vgl. Hagen \& Simonson 2017: 315). $\mathrm{Zu}$ fragen ist, ob attraktivere Funktionen für Frauen in Organisationen zusammen mit einer familienfreundlicheren Gestaltung das ehrenamtliche Engagement der Frauen im Lebensverlauf erhöhen würden. Die spezifische Förderung von Chancen im Ehrenamt von Frauen sollte deshalb nicht auf die Lebensphase Alter begrenzt sein, sondern schon in Jugend- und mittleren Erwachsenenalter gezielt erfolgen.

\section{Literatur}

Abraham, K. G., Helms, S., \& Presser, S. (2009). How social processes distort measurement: The impact of survey nonresponse on estimates of volunteer work in the United States. American Journal of Sociology, 114(4), 1129-1165.

Alisch, M., Ritter, M., Glaser, R., \& Rubin, Y. (2018). Engagement im Bürgerhilfeverein als Balanceakt zwischen sinnvoller Freizeitgestaltung, sozialer Teilhabe und Selbstprofessionalisierung. In: S. Scherger \& C. Vogel (Hrsg.) Arbeit im Alter. Zur Bedeutung bezahlter und unbezahlter Tätigkeiten in der Lebensphase Ruhestand (S. 245-267). Wiesbaden: Springer VS.

Hagen, C., \& Simonson, J. (2017). Inhaltliche Ausgestaltung und Leitungsfunktionen im freiwilligen Engagement. In: J. Simonson, C. Vogel \& C. Tesch-Römer (Hrsg.) Freiwilliges Engagement in Deutschland. Der Deutsche Freiwilligensurvey 2014 (S. 299-331). Wiesbaden: Springer VS.

Künemund, H. (2006). Tätigkeiten und Engagement im Ruhestand. In: C. Tesch-Römer, H. Engstler \& S. Wurm (Hrsg.) Altwerden in Deutschland (S. 289-327). Wiesbaden: VS Verlag für Sozialwissenschaften.

Künemund, H., \& Schupp, J. (2008). Konjunkturen des Ehrenamts - Diskurse und Empirie. In: M. Erlinghagen \& K. Hank (Hrsg.) Produktives Altern und informelle Arbeit in modernen Gesellschaften (S. 145163). Wiesbaden: VS Verlag für Sozialwissenschaften.

Künemund, H., \& Vogel, C. (2018). Altersgrenzen. Theoretische Überlegungen und empirische Befunde zur Beendigung von Erwerbsarbeit und Ehrenamt. In: S. Scherger \& C. Vogel (Hrsg.) Arbeit im Alter. Zur Bedeutung bezahlter und unbezahlter Tätigkeiten in der Lebensphase Ruhestand (S. 75-98). Wiesbaden: Springer VS.

Maurer, S. (2018). Der Einfluss der früheren Erwerbstätigkeit auf freiwilliges Engagement im Ruhestand. In: S. Scherger \& C. Vogel (Hrsg.) Arbeit im Alter. Zur Bedeutung bezahlter und unbezahlter Tätigkeiten in der Lebensphase Ruhestand (S. 195-215). Wiesbaden: Springer VS.

Müller, D., \& Tesch-Römer, C. (2017). Früheres Engagement und Engagementbereitschaft. In: J. Simonson, C. Vogel \& C. Tesch-Römer (Hrsg.) Freiwilliges Engagement in Deutschland. Der Deutsche Freiwilligensurvey 2014 (S. 153-178). Wiesbaden: Springer VS.

Naumann, D., \& Romeu Gordo, L. (2010). Gesellschaftliche Partizipation: Erwerbstätigkeit, Ehrenamt und Bildung. In: A. Motel-Klingebiel, S. Wurm \& C. Tesch-Römer (Hrsg.) Altern im Wandel. Befunde des Deutschen Alterssurveys (DEAS) (S. 118-141). Stuttgart: Kohlhammer. 
Şaka, B. (2018). Einfluss der Kohortenzugehörigkeit auf das ehrenamtliche Engagement im Alter ab 50 Jahren. In: S. Scherger \& C. Vogel (Hrsg.) Arbeit im Alter. Zur Bedeutung bezahlter und unbezahlter Tätigkeiten in der Lebensphase Ruhestand (S. 269-293). Wiesbaden: Springer VS.

Simonson, J., \& Hameister, N. (2017). Sozioökonomischer Status und freiwilliges Engagement. In: J. Simonson, C. Vogel \& C. Tesch-Römer (Hrsg.) Freiwilliges Engagement in Deutschland. Der Deutsche Freiwilligensurvey 2014 (S. 439-464). Wiesbaden: Springer VS.

Simonson, J., \& Vogel, C. (2018/im Erscheinen). Freiwilliges Engagement im Alter. In: K. R. Schroeter, C. Vogel \& H. Künemund (Hrsg.) Handbuch Soziologie des Alter(n)s. Wiesbaden: Springer VS.

Statistisches Bundesamt (2018). Bildungsstand der Bevölkerung - Ergebnisse des Mikrozensus 2017. Wiesbaden: Statistisches Bundesamt.

Vogel, C., Hagen, C., Simonson, J., \& Tesch-Römer, C. (2017). Freiwilliges Engagement und öffentliche gemeinschaftliche Aktivität. In: J. Simonson, C. Vogel \& C. Tesch-Römer (Hrsg.) Freiwilliges Engagement in Deutschland. Der Deutsche Freiwilligensurvey 2014 (S. 91-150). Wiesbaden: Springer VS.

Wetzel, M., \& Simonson, J. (2017). Engagiert bis ins hohe Alter? Organisationsgebundenes ehrenamtliches Engagement in der zweiten Lebenshälfte. In: K. Mahne, J. K. Wolff, J. Simonson \& C. Tesch-Römer (Hrsg.) Altern im Wandel. Zwei Jahrzehnte Deutscher Alterssurvey (DEAS) (S. 81-95). Wiesbaden: Springer VS.

Open Access Dieses Kapitel wird unter der Creative Commons Namensnennung 4.0 International Lizenz (http:// creativecommons.org/licenses/by/4.0/deed.de) veröffentlicht, welche die Nutzung, Vervielfältigung, Bearbeitung, Verbreitung und Wiedergabe in jeglichem Medium und Format erlaubt, sofern Sie den/die ursprünglichen Autor(en) und die Quelle ordnungsgemäß nennen, einen Link zur Creative Commons Lizenz beifügen und angeben, ob Änderungen vorgenommen wurden.

Die in diesem Kapitel enthaltenen Bilder und sonstiges Drittmaterial unterliegen ebenfalls der genannten Creative Commons Lizenz, sofern sich aus der Abbildungslegende nichts anderes ergibt. Sofern das betreffende Material nicht unter der genannten Creative Commons Lizenz steht und die betreffende Handlung nicht nach gesetzlichen Vorschriften erlaubt ist, ist für die oben aufgeführten Weiterverwendungen des Materials die Einwilligung des jeweiligen Rechteinhabers einzuholen.

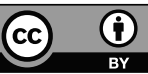

